ARTICLE

Received 30 Apr 2014 | Accepted 6 Mar 2015 | Published 21 Apr $2015 \quad$ DOl: 10.1038/ncomms7873 OPEN

\title{
Live-cell imaging of actin dynamics reveals mechanisms of stereocilia length regulation in the inner ear
}

Meghan C. Drummond ${ }^{1}$, Melanie Barzik ${ }^{1, \star}$, Jonathan E. Bird ${ }^{1, \star}$, Duan-Sun Zhang ${ }^{2}$, Claude P. Lechene ${ }^{3,4}$, David P. Corey ${ }^{2}$, Lisa L. Cunningham ${ }^{5} \&$ Thomas B. Friedman ${ }^{1}$

The maintenance of sensory hair cell stereocilia is critical for lifelong hearing; however, mechanisms of structural homeostasis remain poorly understood. Conflicting models propose that stereocilia F-actin cores are either continually renewed every $24-48 \mathrm{~h}$ via a treadmill or are stable, exceptionally long-lived structures. Here to distinguish between these models, we perform an unbiased survey of stereocilia actin dynamics in more than 500 utricle hair cells. Live-imaging EGFP- $\beta$-actin or dendra2- $\beta$-actin reveal stable F-actin cores with turnover and elongation restricted to stereocilia tips. Fixed-cell microscopy of wild-type and mutant $\beta$-actin demonstrates that incorporation of actin monomers into filaments is required for localization to stereocilia tips. Multi-isotope imaging mass spectrometry and live imaging of single differentiating hair cells capture stereociliogenesis and explain uniform incorporation of ${ }^{15} \mathrm{~N}$-labelled protein and EGFP- $\beta$-actin into nascent stereocilia. Collectively, our analyses support a model in which stereocilia actin cores are stable structures that incorporate new F-actin only at the distal tips.

\footnotetext{
${ }^{1}$ Laboratory of Molecular Genetics, Section on Human Genetics, National Institute on Deafness and Other Communication Disorders, National Institutes of Health, Bethesda, Maryland 20892, USA. ${ }^{2}$ Department of Neurobiology, Harvard Medical School and Howard Hughes Medical Institute, Boston, Massachusetts 02115, USA. ${ }^{3}$ National Resource for Imaging Mass Spectrometry, Brigham and Women's Hospital and Harvard Medical School, Cambridge, Massachusetts 02139, USA. ${ }^{4}$ Division of Genetics, Department of Medicine, Brigham and Women's Hospital and Harvard Medical School, Cambridge, Massachusetts 02139, USA. ${ }^{5}$ Section on Sensory Cell Biology, National Institute on Deafness and Other Communication Disorders, National Institutes of Health, Bethesda, Maryland 20892, USA. * These authors contributed equally to this work. Correspondence and requests for materials should be addressed to T.B.F. (email: friedman@nidcd.nih.gov).
} 
$\mathrm{H}$ air cells of the inner ear transduce sound energy and head movement into afferent nerve signals that are transmitted to the brain. Hair cells owe their name to the staircaseshaped bundle of mechanosensory stereocilia (Fig. 1), which are actin-based structures that project from the apical surface into the potassium-rich endolymph of the cochlear duct and the vestibular labyrinth. These mechanosensitive cells are terminally differentiated in mammals and are not regenerated when they $\mathrm{die}^{4,5}$.

The stereocilia bundle develops from a patch of microvilli on the apical surface of a differentiating hair cell. Each microvillus undergoes a complex process of programmed elongation and thickening by increasing the length and number of polarized parallel actin filaments (F-actin) that form the paracrystalline core of each stereocilium ${ }^{1,2,6}$. The barbed (plus) ends of the actin filaments within each core terminate near the distal tip, which is presumed to be the site of monomer (G-actin) addition during stereocilia development ${ }^{3}$. Conversely, the pointed (minus) ends of the filaments terminate near the apical surface of the hair cell or perhaps extend into the rootlets that anchor stereocilia in the cuticular plate ${ }^{3,7,8}$. Several actin crosslinking proteins including espin, plastin/fimbrin, fascin-2 and TRIOBP contribute to the rigidity of the $\mathrm{F}$-actin cores in stereocilia ${ }^{8-12}$. The staircase architecture (Fig. 1) and stiffness of the stereocilia bundle are crucial for the exquisite sensitivity of hair cells to mechanical displacements.

In contrast to the dynamic nature of microvilli, which are continuously created and disassembled with a half-life of a few minutes $^{13}$, mammalian stereocilia are proposed to last for the lifetime of a hair cell. Evidence from transgenic mice suggests that once irreparably damaged, individual stereocilia are resorbed by the hair cell and not replaced ${ }^{14,15}$. It follows that the stereocilia actin cores of post-mitotic hair cells must be precisely maintained throughout the life of the organism. However, the molecular mechanisms that preserve these crucial mechanosensitive organelles are largely uncharacterized.

One intriguing model to explain the longevity of stereocilia is that their F-actin cores are continuously renewed via a perpetual actin treadmill. This model requires that actin monomer removal (depolymerization) at the base occurs at exactly the same rate as actin monomer addition (polymerization) at the distal tip in order to strictly maintain the steady-state length of each stereocilium. In this rapid turnover (treadmill) model, complete turnover of the F-actin core occurs every $24-48 \mathrm{~h}^{16-18}$. Actin treadmills are well studied in vitro ${ }^{19,20}$ and in microvilli, lamellipodia and dendritic spines in vivo ${ }^{21-23}$. Although actin turnover in stereocilia has been mathematically modelled $^{24}$, direct experimental measures of polymerization and depolymerization rates have not been reported. Retrograde flow of newly incorporated and recycled G-actin in stereocilia cores was inferred from individual static images of paraformaldehydefixed neonatal hair cells at various time points following viral or biolistic gene gun transfection (propulsion of gold particles coated with plasmid DNA) expressing either enhanced green fluorescent protein (EGFP)- $\beta$-actin or EGFP-espin ${ }^{16-18}$.
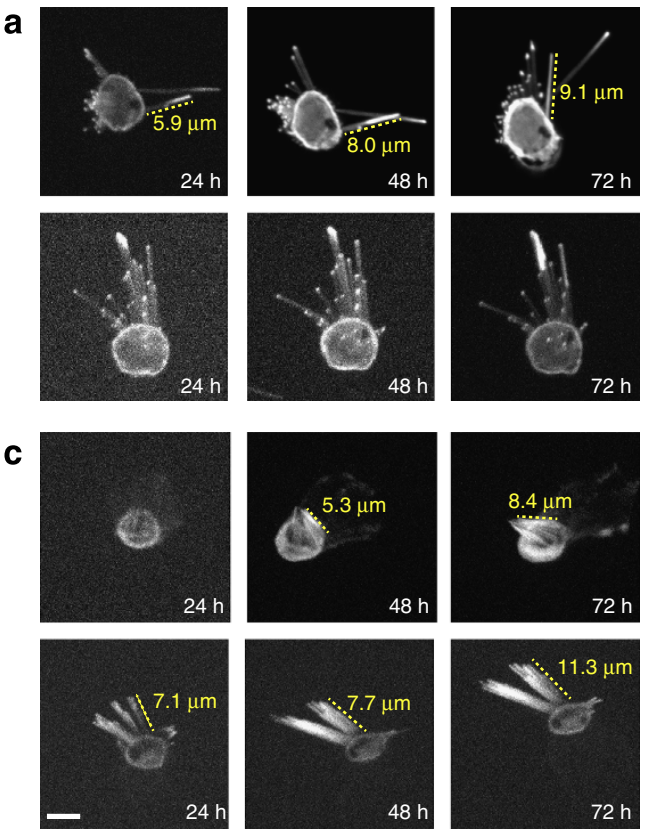

b

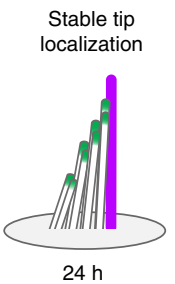

ronous
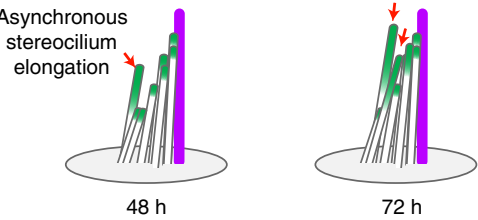

$72 \mathrm{~h}$

\section{d}

Stereociliogenesis
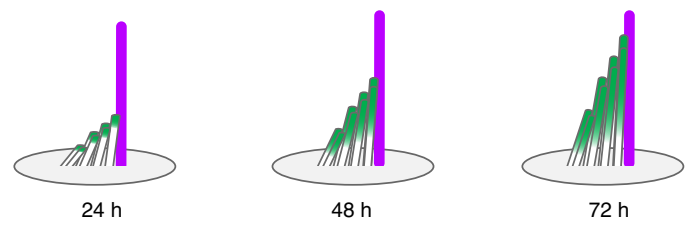

e

Treadmill model
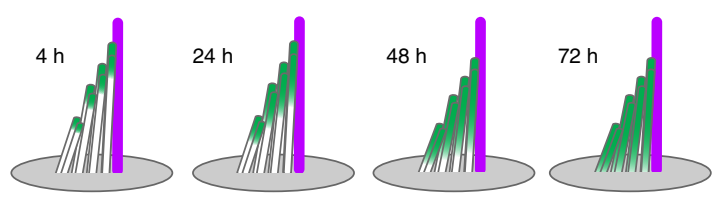

Figure 1 | Live-cell imaging reveals different classes of EGFP-p-actin dynamics in hair cell stereocilia. (a) Still frames and (b) schematic representations of stereocilia bundles demonstrating steady-state tip localization of EGFP- $\beta$-actin (green) and asynchronous elongation (red arrow). The majority of stereocilia in the bundle retained stable tip localization of EGFP- $\beta$-actin throughout the live-imaging experiment. The lengths of stereocilia with asynchronous elongation (a) (yellow dashed line) were measured and are shown in yellow at each time point. (c) Stereociliogenesis of nascent stereocilia bundles on developing hair cells is shown in still frames and (d) illustrated in a schematic. Over $72 \mathrm{~h}$ of live imaging, stereocilia lengthen (yellow dashed lines). (e) Schematic representing the stereociliar F-actin treadmill hypothesis ${ }^{1-3}$. All length changes were measured in 3D using Volocity. Kinocilia are illustrated in purple. All 112 movies are available in Supplementary Movies 1-6. Scale bar, $5 \mu \mathrm{m}$. 
In contrast to the rapid turnover model, the interpretation of data from other experiments indicates that stereocilia cores undergo slow protein turnover, on the order of weeks to months; a concept consistent with stereocilia cores containing exceptionally long-lived proteins (ELLPs) ${ }^{25}$. This model is supported by experiments in adult mice using multi-isotope imaging mass spectrometry (MIMS, measuring ${ }^{15} \mathrm{~N}$-leucine incorporation) and conditional mouse mutants in which $A c t b$, encoding $\beta$-actin, expression is controlled by tamoxifen-inducible cre recombinase ${ }^{26}$. These data demonstrated a 'hotspot' of protein turnover that was restricted exclusively to the distal tip compartment in stereocilia ${ }^{26}$. Similar to the experimental evidence interpreted as supporting a rapid turnover model, the slow turnover model was inferred from a collection of individual static images of fixed hair cells from different animals at various time points.

In this report, we use continuous live-cell imaging in single hair cells to resolve these conflicting models. In addition, we provide MIMS data and high-resolution confocal images of over 400 stereocilia bundles from fixed hair cells transfected with EGFP- $\beta$ actin or with mutant forms of EGFP- $\beta$-actin that are unable to polymerize into filaments. Our data show that only actin in the distal tips of stereocilia is rapidly renewed and polymerization dependent, while stereocilia cores are stable structures.

\section{Results}

Visualization of EGFP- $\beta$-actin in living hair cells. We hypothesized that live-cell imaging of hair cells biolistically transfected (that is, by gene gun) with EGFP- $\beta$-actin would enable us to collect longitudinal data from individual cells and would therefore reveal actin dynamics that cannot be observed using fixed samples. Given that utricular hair cells have longer stereocilia bundles, a higher transfection efficiency by gene gun, and were used for previous studies ${ }^{16-18,26}$ we chose to image utricle explant cultures. In three independent experiments, spinning-disk confocal microscopy was used to record actin dynamics in 14 transfected hair cells that displayed EGFP- $\beta$-actin localization at the tips of stereocilia (Supplementary Movie 1). We reasoned that cells exhibiting tip localization of EGFP- $\beta$-actin would offer the maximum potential to observe rapid turnover of the F-actin cores postulated to occur in the treadmill model. In the majority of stereocilia after a minimum of $62 \mathrm{~h}$ of continuous live imaging, we did not detect movement of EGFP- $\beta$-actin from the tips towards the bases, as would be expected if a treadmill mechanism existed. Occasionally, a single stereocilium within a bundle displayed activity consistent with an F-actin treadmill; however, these unusual stereocilia were rare (Supplementary Movie 1). Instead, we consistently captured two distinct phenomena: (1) steady-state localization of EGFP- $\beta$ actin in the distal stereocilia tips; and (2) asynchronous elongation of stereocilia (Fig. 1). In these cells, steady-state localization of EGFP- $\beta$-actin at stereocilia tips continued for up to $48 \mathrm{~h}$. Beyond $48 \mathrm{~h}$, elongation of some stereocilia within the bundle was recorded (Fig. 1a and Supplementary Movie 1).

Expression of EGFP- $\beta$-actin during our live-cell imaging experiments was under the control of a cytomegalovirus (CMV) promoter. We considered the possibility that overexpression of EGFP- $\beta$-actin resulted in a constant increase in fluorescence intensity at stereocilia tips, causing a corresponding increase in dynamic range that might mask lower levels of incorporation within the F-actin core of the stereocilium shaft. If newly synthesized EGFP- $\beta$-actin is integrated into the cores of stereocilia in a manner consistent with a treadmill, then one would expect to observe a declining gradient of EGFP fluorescence from the tips towards the bases of stereocilia. To address this possibility, we performed line scans of stereocilia from three-dimensional (3D)-volume reconstructed bundles
(Fig. 2; Supplementary Fig. 1). The fluorescence intensity in the cuticular plate and distal tip compartment increased over time; however, we did not detect a corresponding increase or gradient of EGFP- $\beta$-actin along the length of the stereocilium shaft. We did detect a marginal, but uniform increase in fluorescence intensity along the length of the stereocilium that may represent a steady-state level of actin peripheral to the F-actin core (Fig. 2b). These data suggest that any incorporation of EGFP- $\beta$-actin into the F-actin core from the tips consistent with an F-actin treadmill is below the limits of detection.

Live imaging captures stereociliogenesis. While imaging the mouse utricular hair cells described above, we also captured de novo formation of stereocilia on the apical surfaces of seven EGFP- $\beta$-actin-expressing, newly differentiating hair cells. As the time-lapse experiment proceeded, the nascent stereocilia on these seven cells matured from microvilli and fluoresced green uniformly along their entire length consistent with the expression of EGFP- $\beta$-actin from the initiation of bundle formation (Fig. 1b and Supplementary Movies 2 and 3). To examine the extent to which stereociliogenesis occurs in the neonatal utricle, we performed a second set of three independent experiments in which we imaged every EGFP- $\beta$-actin-transfected cell in each utricle culture. The plasmid-coated gold particles used in gene gun transfection are $1 \mu \mathrm{m}$ in diameter and are expected to periodically strike and damage stereocilia bundles (Fig. 3a). Therefore, hair cells in which stereocilia were fused, bent or missing $(39 / 112)$ were excluded from the analyses (Fig. 3b-d, Table 1, Supplementary Movies 4-6). While actin dynamics at the tips of stereocilia may be influenced by a functional MET channel ${ }^{27}$, the F-actin core is not known to be directly affected by the presence of tip links, and therefore splayed bundles were included in the analyses. We found that stereocilia bundles in these additional 73 EGFP- $\beta$-actin-transfected hair cells all demonstrated one of the following two types of actin dynamics: steady-state tip localization with or without asynchronous elongation, or stereociliogenesis. Therefore, out of a total of 73 cells over six independent experiments, EGFP- $\beta$-actin remained steady state at the tips of morphologically stable stereocilia with or without asynchronous elongation in $53 \%$ of cells (29/73). Stereociliogenesis was observed in $60 \%$ of cells (44/73; Table 1), consistent with data indicating that $50 \%$ of mouse utricular hair cells differentiate and develop hair bundles during the first two weeks of postnatal development ${ }^{28,29}$. All of these live-imaging movies are available for viewing (Supplementary Movies 1-6).

EGFP- $\beta$-actin in stereocilia of fixed hair cells. Given that our data obtained from live-cell imaging are in direct opposition to previously published static, fixed-cell images ${ }^{16-18}$, we also performed fixed-cell confocal imaging of EGFP- $\beta$-actinexpressing hair cells. While live-cell imaging has the advantage of obtaining longitudinal data from individual cells, laser excitation intensity must be delicately balanced to limit EGFPphotobleaching and the generation of radical oxygen species. Confocal microscopy of fixed cells allows for the use of greater laser excitation intensity and a phalloidin counterstain to visualize the F-actin cores of stereocilia, and can yield images with higher resolution because the mounting media is optically matched to the objective lens. Similar to data we obtained with live-cell imaging, 49\% (50/103) of biolistically transfected stereocilia bundles showed evidence of damage and were excluded from further analysis. We observed tip localization of EGFP- $\beta$-actin in $100 \%(14 / 14)$ of undamaged transfected stereocilia bundles $4 \mathrm{~h}$ post transfection (Fig. 4a, Table 1), consistent with previously published data ${ }^{16-18}$. However, in contrast to previous reports, we 
a
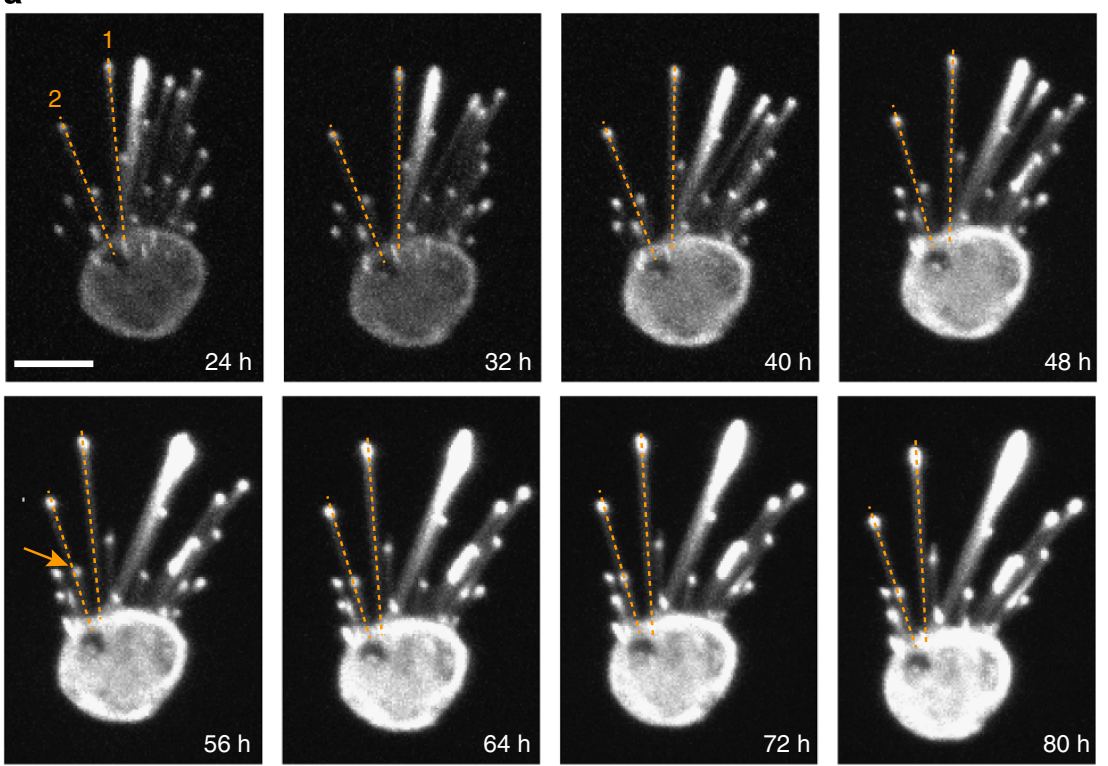

b
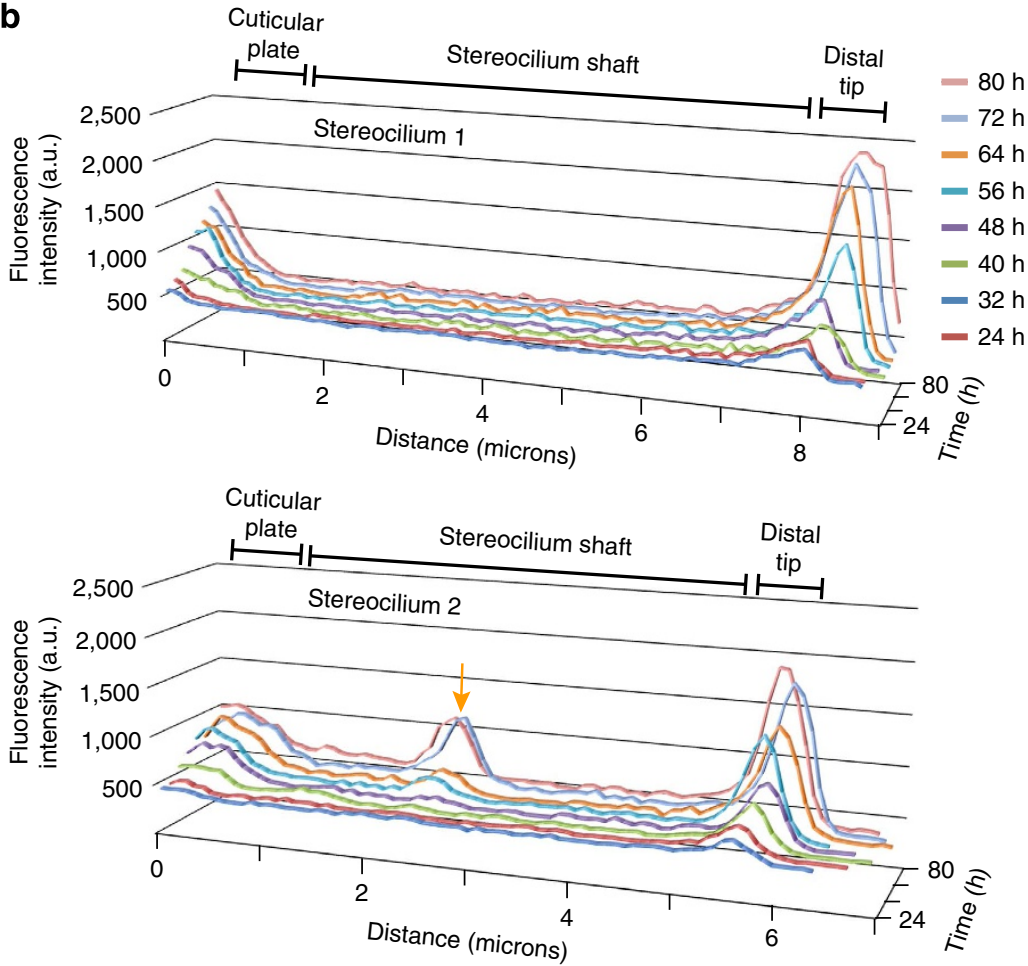

Figure 2 | Quantification of intensity profiles along the lengths of stereocilia. (a) Still frames from a live-imaged hair cell with stable localization of EGFP- $\beta$-actin in the distal tip compartment. Dashed lines (orange) indicate stereocilia that were line-traced every $8 \mathrm{~h}$ beginning $24 \mathrm{~h}$ post transfection with pEGFP- $\beta$-actin. Images without line traces are available in Supplementary Fig. 1. (b) Line traces from stereocilia 1 and 2 demonstrate increasing levels of EGFP- $\beta$-actin in the cuticular plate and distal tip compartment, but not along the stereocilia shaft and confirm steady-state tip localization. Over the course of the experiment, additional stereocilia move in close proximity of stereocilium 2 (arrows) (a), resulting in extra peaks in the line traces that correspond to the distal tip of the second stereocilium. Scale bar, $5 \mu \mathrm{m}$.

found that cells expressing EGFP- $\beta$-actin imaged $24 \mathrm{~h}$ post transfection displayed variability in the localization of the fluorescent signal. Specifically, 55\% of morphologically intact transfected cells (22/40) still had EGFP- $\beta$-actin enriched at the tips of the stereocilia bundle, with weak uniform labelling of stereocilia shafts, whereas the remaining 45\% (18/40) of bundles had uniformly strong labelling of stereocilia (Fig. 4a, Table 1). These data are consistent with our live-imaging data and support our conclusions that EGFP- $\beta$-actin dynamics in hair cell stereocilia fall into two distinct categories: (1) enrichment at tips with or without asynchronous elongation; and (2) fully labelled stereocilia bundles.

Actin polymerization is necessary for tip localization. To investigate whether weak uniform EGFP- $\beta$-actin labelling along 
a

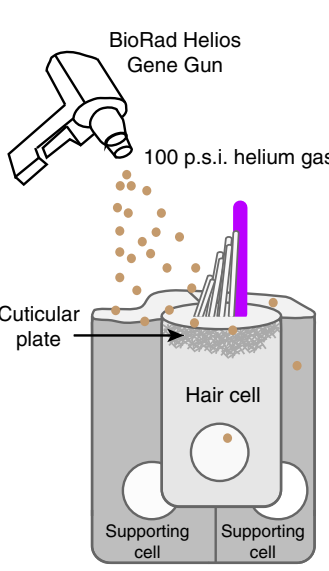

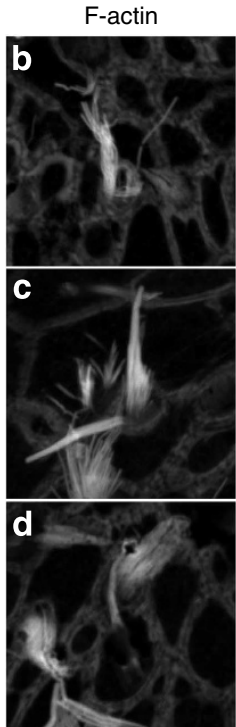
EGFP- $\beta$-actin $\quad F$-actin EGFP- $\beta$-actin

Phase contrast

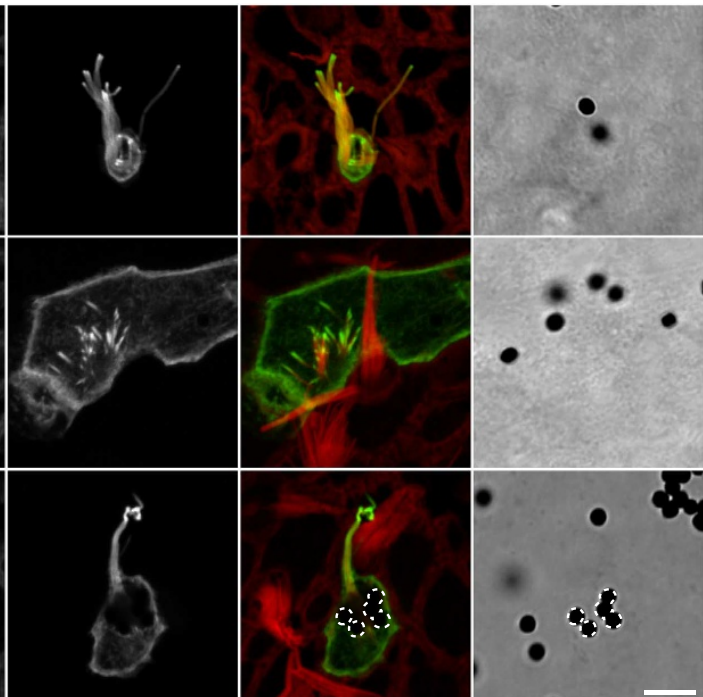

Figure 3 | Biolistic gene gun transfection may result in damage to hair cells. (a) Penetration of the $1 \mu \mathrm{m}$ gold DNA-coated particles through the cell membrane and cuticular plate of hair cells results in successful transfection of plasmid DNA; however, bullets can strike and damage stereocilia bundles in the process. (b-d) Representative images of stereocilia bundles classified as damaged and excluded from further analyses. Cells were biolistically transfected with EGFP- $\beta$-actin (green), fixed and counterstained with rhodamine phalloidin (red) to visualize all F-actin. Phase contrast shows positions of gold bullets. Dotted lines indicate gold particles on the surface of a hair cell. Scale bar, $5 \mu \mathrm{m}$.

Table 1 | EGFP localization in sensory hair cells.

\begin{tabular}{|c|c|c|c|c|c|c|c|}
\hline & $\begin{array}{c}\text { Time after } \\
\text { transfection }(h)\end{array}$ & $\begin{array}{l}\text { Transfected } \\
\text { cells }\end{array}$ & $\begin{array}{l}\text { Damaged } \\
\text { cells }^{\star}\end{array}$ & $\begin{array}{c}\text { Cells included in } \\
\text { analysis }\end{array}$ & $\begin{array}{l}\text { Stereocilia } \\
\text { tips }\end{array}$ & $\begin{array}{l}\text { Uniformly labelled } \\
\text { stereocilia }\end{array}$ & $\begin{array}{c}\text { Primarily cell body, weakly } \\
\text { labelled stereocilia }\end{array}$ \\
\hline \multirow[t]{3}{*}{ EGFP- $\beta$-actin } & $\begin{array}{c}24-96^{\dagger} \\
\text { (live imaged) }\end{array}$ & 112 & $38(34 \%)$ & 73 & $29(40 \%)$ & $44(60 \%)$ & 0 \\
\hline & 4 & 21 & $7(33 \%)$ & 14 & $14(100 \%)$ & 0 & 0 \\
\hline & 24 & 82 & $43(52 \%)$ & 40 & $22(55 \%)$ & $18(45 \%)$ & 0 \\
\hline \multirow[t]{2}{*}{ EGFP (control) } & 4 & 48 & $12(25 \%)$ & 36 & 0 & 0 & $36(100 \%)$ \\
\hline & 24 & 46 & $17(37 \%)$ & 29 & 0 & 0 & $29(100 \%)$ \\
\hline \multirow[t]{2}{*}{ EGFP- $\beta$-actin G63D } & 4 & 21 & $6(29 \%)$ & 15 & $1(7 \%)$ & $8(53 \%)$ & $6(40 \%)$ \\
\hline & 24 & 143 & $68(48 \%)$ & 75 & $5(7 \%)$ & $22(29 \%)$ & $48(48 \%)$ \\
\hline \multirow[t]{2}{*}{ EGFP- $\beta$-actin G13R } & 4 & 6 & $2(33 \%)$ & 4 & 0 & 0 & $4(100 \%)$ \\
\hline & 24 & 60 & $24(40 \%)$ & 36 & 0 & 0 & $36(100 \%)$ \\
\hline Total & & 539 & $217(40 \%)$ & 322 & 72 & 92 & 159 \\
\hline
\end{tabular}

EGFP, enhanced green fluorescent protein.

${ }^{\star}$ Cells classified as damaged were not included in further analyses.

$\dagger 96 \mathrm{~h}$ was the maximum duration of live imaging.

the lengths of stereocilia could be explained by diffusion from the cell body into the stereocilia bundle, we transfected neonatal utricle cultures with EGFP- $\beta$-actin mutants (either p.G13R or p.G63D) that fold correctly but are unable to incorporate into filaments ${ }^{30,31}$. We reasoned that EGFP- $\beta$-actin produced in the cell body might diffuse freely into the space surrounding the paracrystalline actin core and into the tip compartment without incorporating into the F-actin core. We transfected hair cells with either wild-type $\beta$ actin, mutant $\beta$-actin or an EGFP-only negative control and imaged hair cells fixed at 4 and $24 \mathrm{~h}$ post transfection. Unlike wildtype EGFP- $\beta$-actin, we did not observe accumulation of mutant EGFP- $\beta$-actin ${ }^{\mathrm{G} 13 \mathrm{R}}$ or EGFP- $\beta$-actin ${ }^{\mathrm{G} 63 \mathrm{D}}$ at stereocilia tips in the vast majority (124/130) of fixed hair cells at 4 and $24 \mathrm{~h}$ post transfection (Fig. 4c,d, Table 1). However, EGFP- $\beta$-actin ${ }^{\text {G13R }}$ or EGFP- $\beta$-actin ${ }^{G 3 D}$ was present in the stereocilia shaft at a similar level observed for wild-type EGFP- $\beta$-actin. In 7\% (6/90) of hair cells transfected with EGFP- $\beta$-actin ${ }^{\mathrm{G} 63 \mathrm{D}}$, we did detect enrichment of EGFP fluorescence at the tips of stereocilia that we did not observe in EGFP- $\beta$-actin ${ }^{\text {G13R }}$-transfected hair cells. These data are consistent with published biochemical data demonstrating that EGFP- $\beta$-actin ${ }^{G 13 R}$ is incapable of polymerizing into filaments in the presence of wild-type $\beta$-actin ${ }^{3}$, while $\beta$-actin ${ }^{\mathrm{G} 63 \mathrm{D}}$ can form small filaments in the presence of a high concentration of wild-type $\beta$-actin ${ }^{30}$. These data demonstrate that incorporation of actin monomers into filaments is required for trafficking of EGFP- $\beta$ actin to the tips of hair cell stereocilia, retention of EGFP- $\beta$-actin at the tips or both. Control experiments in which hair cells were transfected with EGFP only (that is, no actin) demonstrated that complete fluorescent labelling of stereocilia shafts can be achieved solely by diffusion of proteins from the cell body (Fig. 4b, Table 1). Thus, our data indicate that some macromolecules in the cell body are not restricted from diffusion into the stereocilia bundle compartment.

Photoconversion of dendra2- $\beta$-actin at stereocilia tips. To further investigate the dynamics of $\beta$-actin at the tips of stereocilia, we biolistically transfected a plasmid encoding 


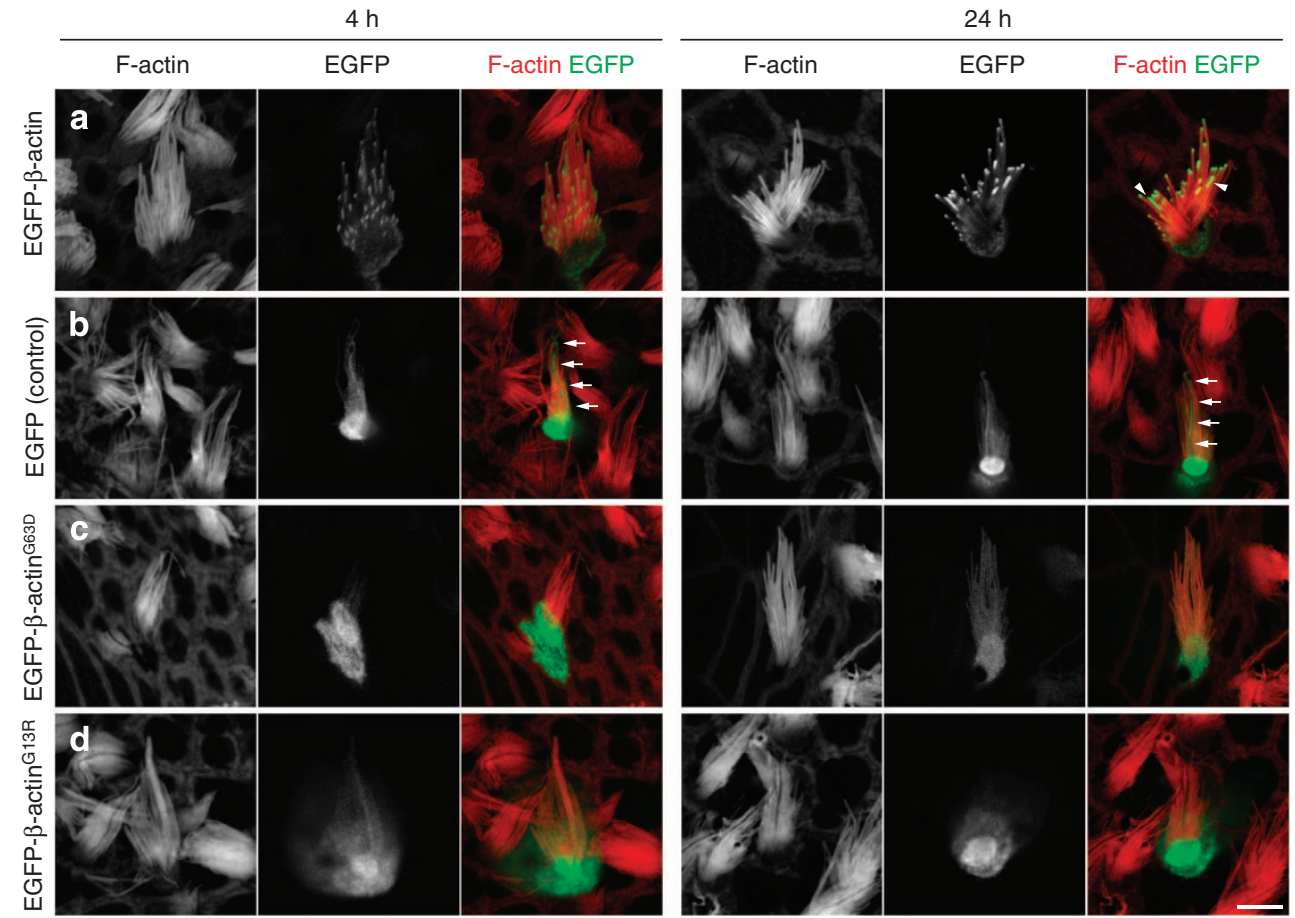

Figure 4 | Localization of EGFP- $\beta$-actin at stereocilia tips is polymerization dependent. Localization of wild-type EGFP- $\beta$-actin, EGFP alone (negative control), and mutant EGFP- $\beta$-actin ${ }^{\text {G63D }}$ or mutant EGFP- $\beta$-actin ${ }^{\text {G13R }}$ (green) at 4 and 24 h post transfection. (a) Wild-type EGFP- $\beta$-actin was enriched at stereocilia tips with diffuse labelling along the shafts at 4 and $24 \mathrm{~h}$ post transfection. Consistent with our live-cell imaging observations, two stereocilia appear to have elongated from the distal end (arrowheads). In contrast, (b-d) EGFP- $\beta$-actin G63D, EGFP- $\beta$-actin ${ }^{\text {G13R }}$ and the EFGP control were present diffusely throughout the hair cell body, stereocilia bundle and occasionally the kinocilium (arrows) at 4 and $24 \mathrm{~h}$. F-actin is labelled with rhodamine phalloidin (red). Scale bar, $5 \mu \mathrm{m}$.

dendra2- $\beta$-actin into cultured neonatal utricles. Dendra2 is a monomeric green fluorescent molecule that can be irreversibly converted to emit red fluorescence by exposure to $405-\mathrm{nm}$ (and to a lesser extent $488 \mathrm{~nm}$ ) light. The red variant of the molecule is photo-stable, making it possible to image a population of photoconverted actin molecules for several hours ${ }^{32}$. We used photoconversion to track the movement of a specific population of differentially labelled fluorescent actin.

Locations within dendra2- $\beta$-actin-expressing hair cells were specifically targeted with a $405 \mathrm{~nm}$ laser to photoconvert dendra2$\beta$-actin from green (dendra2- $\beta$-actin GREEN) to red (dendra2- $\beta$ actin $^{R E D}$ ). Optical sections through the entire hair cell were taken every $30 \mathrm{~min}$ for $24 \mathrm{~h}$. The dynamics of photoconverted dendra2$\beta$-actin ${ }^{\text {RED }}$ were analysed using two independent methods (Fig. 5). We used Volocity 3D image analysis software to generate volume-rendered 3D images. Measurements were made at 6-h intervals to determine (1) the distance from the tip of a stereocilium to its insertion point in the hair cell cuticular plate and (2) the distance from the lower boundary of the photoconverted population of actin to the insertion point in the cuticular plate (Fig. 5). Using these methods, we found that the position of the photoconverted population of dendra2- $\beta$-actin ${ }^{\text {RED }}$ remained stable relative to the stereocilium insertion point, while the total length of the stereocilium increased through addition of non-photoconverted dendra2- $\beta$-actin GREEN distal to the photoconverted dendra2- $\beta$-actin ${ }^{\text {RED }}$ (Fig. 5, Supplementary Movie 7). To avoid any observer bias in these direct measurements, we also utilized Volocity to generate an automated method of defining and quantifying the distance from the photoconverted dendra2$\beta$-actin ${ }^{\text {RED }}$ to the edge of the cuticular plate, which fluoresced green with non-photoconverted dendra2- $\beta$-actin GREEN (Fig. $5 \mathrm{~d}$ ). In agreement with our direct measurements, the softwaregenerated automated measurements indicate that the position of the photoconverted dendra2- $\beta$-actin ${ }^{\mathrm{RED}}$ remains stable until the point at which the signal is below the limits of detection (usually after about $16 \mathrm{~h}$ of imaging). These data are consistent with our observations using live imaging of EGFP- $\beta$-actin demonstrating that stereocilia can elongate their F-actin cores, but do not treadmill.

Developing hair bundles are detected by MIMS. In addition to live imaging, a second method was used to capture stereociliogenesis in postnatal utricles in vitro. We examined the incorporation of newly synthesized protein into growing stereocilia using $\mathrm{MIMS}^{26}$, a method that reveals the presence of selected isotopes in thin sections of tissue. To label newly synthesized protein with ${ }^{15} \mathrm{~N}$, a rare but stable isotope of nitrogen, we transferred newborn mouse pups to foster mothers whose food contained ${ }^{15} \mathrm{~N}$-leucine. Pups continued to nurse from these ${ }^{15} \mathrm{~N}$ foster mothers for 4 or 15 days before sacrifice, so that protein synthesized after birth would be ${ }^{15} \mathrm{~N}$-labelled. We used MIMS to reveal the localization of both postnatally synthesized protein containing ${ }^{15} \mathrm{~N}$ and prenatally synthesized protein containing ${ }^{14} \mathrm{~N}$, as previously described ${ }^{26}$. The location of postnatally synthesized protein was indicated as the ratio of ${ }^{15} \mathrm{~N} /{ }^{14} \mathrm{~N}$ at every point in the image (Fig. 6). At postnatal day 4, most hair bundles contained relatively low levels of ${ }^{15} \mathrm{~N}$, suggesting that they had developed before birth. However, about a quarter of stereocilia bundles ( 8 out of 34 observed with MIMS) were shorter in length and had higher ${ }^{15} \mathrm{~N}$ levels, suggesting that they had developed postnatally. At postnatal day 15 most bundles had reached a mature length, but nearly half $(20 / 43)$ showed high ${ }^{15} \mathrm{~N}$ incorporation, indicating postnatal development. Thus utricular stereocilia can be labelled along their entire length with labelled protein if they develop after birth. These MIMS data, together 


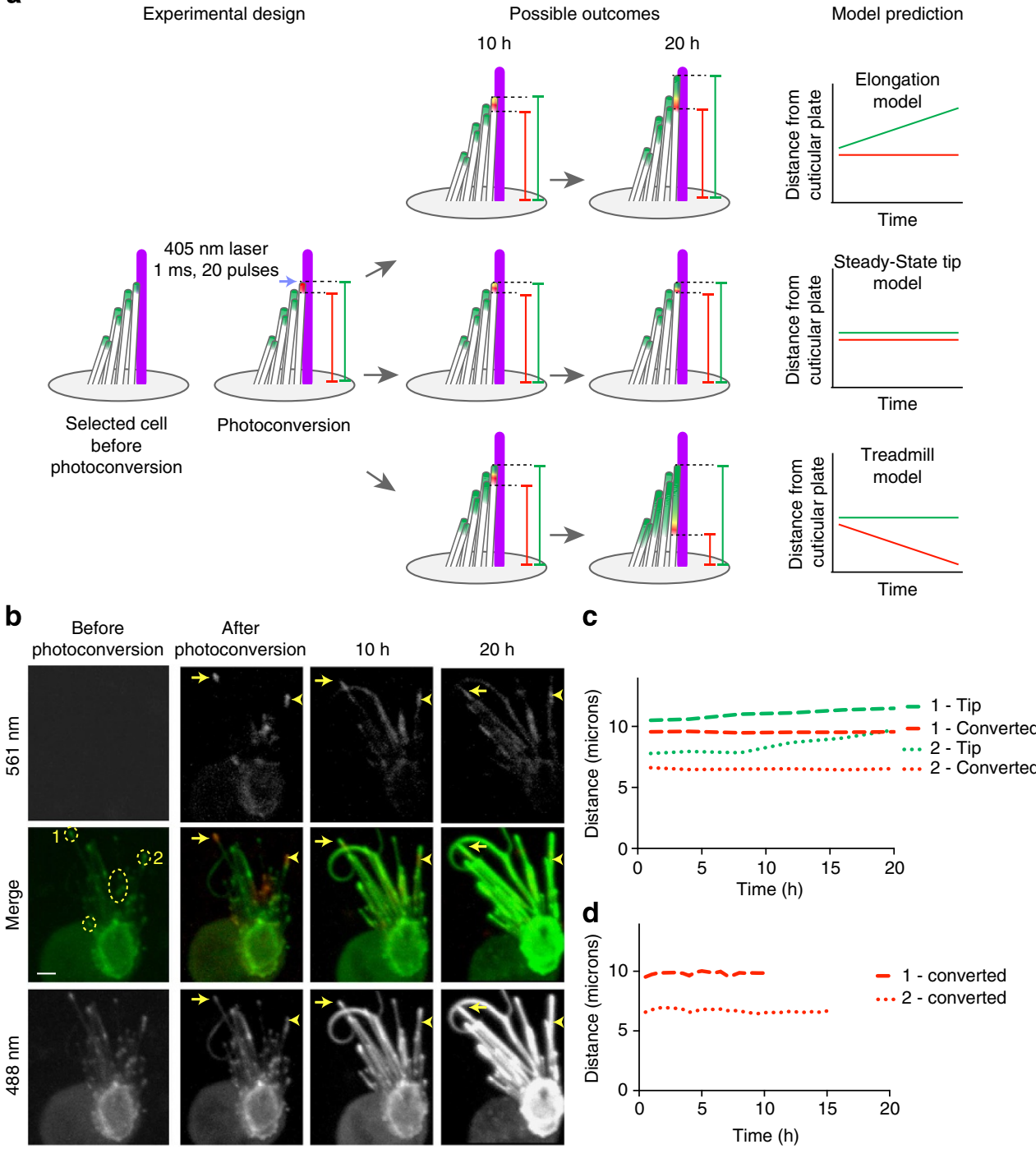

Figure 5 | Photoconversion of dendra2- $\beta$-actin reveals stable actin in stereocilia cores. (a) Schematic of the experimental design, possible outcomes of the experiment and theoretical data of each predicted model. Cultured utricles biolistically transfected with pDendra2- $\beta$-actin were mounted onto a spinning-disk microscope for live imaging. The distal tip compartments of stereocilia were targeted with a $405 \mathrm{~nm}$ laser to photoconvert dendra 2 from green to red. Dashed lines indicate the tip of the stereocilium and the lower boundary of the photoconverted region. (b) Time-lapse images from a representative cell before photoconversion, immediately after photoconversion and at 10 and $20 \mathrm{~h}$ post-photoconversion. Photoconverted regions of interest are denoted by a yellow dashed circles. A $488 \mathrm{~nm}$ laser and $561 \mathrm{~nm}$ laser were used to excite non-photoconverted dendra2- $\beta$-actin (green) and photoconverted dendra2- $\beta$-actin (red), respectively. During photoconversion, dendra2- $\beta$-actin in the cuticular plate was also converted and can be seen moving into the shafts of stereocilia (Supplementary Movie 7). (c) The distance from the boundary of the photoconverted dendra2- $\beta$-actin (red) and the distance from the distal tip of the stereocilium (green) were measured every $8 \mathrm{~h}$ and plotted for stereocilia tips 1 and 2 indicated by the yellow arrow and arrowheads, respectively, in panel $\mathbf{b}$. Measurements were made with both the green and red channels displayed to determine the insertion point of the photoconverted stereocilium into the cuticular plate. The distance from the lower boundary of the photoconverted dendra2- $\beta$-actin to point of insertion of the stereocilium into the apical surface of the hair cell does not change, consistent with steady-state tip localization of photoconverted dendra2- $\beta$-actin. In contrast, the distance from the distal tip to the apical surface increases over time, consistent with stereocilia elongation. (d) Automated measurements were made using Volocity software to plot the distance from the centroid of the photoconverted dendra2- $\beta$-actin to the edge of the non-photoconverted cuticular plate (green). Scale bar, $5 \mu \mathrm{m}$.

with our observations using both EGFP- $\beta$-actin and photoconvertible dendra2- $\beta$-actin, are consistent with a model in which stereocilia F-actin cores, once formed, do not treadmill. Instead, stereocilia are exceptionally long-lived structures with newly synthesized F-actin restricted to the distal tips.

\section{Discussion}

The rapid and slow turnover models for the maintenance of hair cell stereociliar F-actin cores are seemingly irreconcilable ${ }^{16-18,26}$.
Our data from live imaging of hair cells and static images from fixed EGFP- $\beta$-actin-transfected tissue and MIMS show that (a) turnover of F-actin is restricted to the distal stereocilia tip compartment, (b) the accumulation of actin at stereocilia tips requires actin polymerization, and (c) stereociliogenesis, during which nascent bundles elongate and thicken their F-actin cores, results in stereocilia cores labelled along their length with EGFP$\beta$-actin.

The use of live-cell imaging allowed us to longitudinally observe actin dynamics in the same stereocilia bundle and resolve 

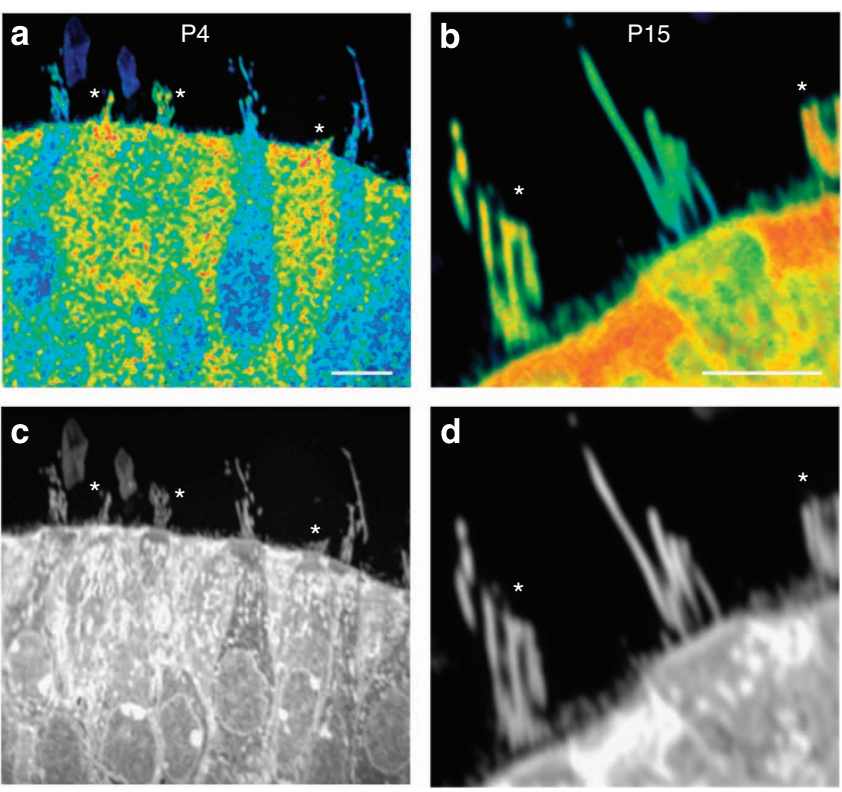

Figure 6 | Postnatal stereociliogenesis is detected with MIMS.

Representative images of mouse utricles at postnatal day 4 (P4) and P15. The ${ }^{15} \mathrm{~N} /{ }^{14} \mathrm{~N}$ ratio is illustrated in pseudocolour, with blue indicating low and red indicating high incorporation of ${ }^{15} \mathrm{~N}$ into newly synthesized proteins. (a) At P4, three of the six stereocilia bundles in the field are small and show high levels of protein incorporation (asterisks); they are consistent with being immature bundles that developed after ${ }^{15} \mathrm{~N}$ feeding began at PO. (b) At P15, bundles are approaching their mature sizes, but one has little incorporation of new protein (centre), whereas two show high incorporation of new protein (asterisks) consistent with development after PO. (c,d) Images of the same fields, indicating total protein. Scale bar, $5 \mu \mathrm{m}$.

several long-standing questions. Had we only observed images of different fixed samples at multiple post-transfection intervals, the rapid turnover stereocilia treadmill model would have been a plausible explanation for our data, given the well-documented role of actin treadmilling in other types of cellular protrusions, such as dendritic spines ${ }^{21,22}$. In fact, when we performed similar fixed-cell experiments (Fig. 4), two patterns of EGFP- $\beta$-actin localizations within stereocilia bundles at 4 and $24 \mathrm{~h}$ post transfection were observed, further underscoring the importance of following EGFP- $\beta$-actin dynamics in the same cell over time by live imaging. As a specific example, it is impossible to distinguish between elongation from the tips of stereocilia and a retrograde actin treadmill unless multiple time points are collected for the same hair cell bundle and $3 \mathrm{D}$ measurements are made.

A few caveats need to be considered when interpreting our data. First, caution must be exercised when assigning meaning to the percentage of cells that show a particular EGFP- $\beta$-actin localization. Of the 539 cells that we imaged, 217 either died shortly after the initiation of the experiment or their stereocilia bundles were extensively damaged by the mechanical trauma of biolistic (gene gun) transfection. Successful biolistic transfection requires that the plasmid-coated $1 \mu \mathrm{m}$ gold particles penetrate the cellular membrane without striking the prominent but fragile stereocilia bundle ${ }^{33}$. Thus, it is not surprising that a significant proportion of cells transfected with this technique are damaged. Moreover, the proportion of cells categorized as 'enriched at tips' versus 'fully labelled bundle' may reflect increased efficiency of transfecting a nascent hair cell compared with that of a mature hair cell. In mature hair cells, the dense, heavily crosslinked
F-actin comprising the cuticular plate at the apical surface (Fig. 3a) is likely to hinder biolistic transfection, whereas the gold particle may more easily penetrate the developing hair cell through an immature cuticular plate. Finally, overexpression of EGFP- $\beta$-actin may itself damage a hair cell, as actin concentrations in cells are closely controlled ${ }^{34}$. In particular, it is unclear whether the observed asynchronous elongation of an individual stereocilium in a bundle is a normal occurrence, a response to damage from biolistic transfection or an artifact of overexpressing EGFP- $\beta$-actin in cultured hair cells. Intravital imaging of hair cells in live animals will be necessary to resolve these open questions.

Data obtained using non-polymerizable missense mutants EGFP- $\beta$-actin ${ }^{\mathrm{G} 13 \mathrm{R}}$ or EGFP- $\beta$-actin ${ }^{\mathrm{G} 63 \mathrm{D}}$ indicate that the actin population in the distal tip compartment of stereocilia is likely composed of F-actin. These data, along with evidence from a companion paper $^{35}$ that the tip compartment is renewed at a faster rate than the actin core, raise questions about the role(s) of this dynamic F-actin pool in this distinct subcellular compartment. In addition to its established functions in cell structure and motility ${ }^{36,37}$, F-actin also serves as a scaffold or barrier to restrict the trafficking of vesicles or molecules ${ }^{38,39}$. F-actin in the distal tip compartment of stereocilia may be an extension of the paracrystalline core or perhaps this distinct population of actin has an alternative role in regulating stereocilia tip dynamics in close proximity to the site of mechanoelectric transduction $^{27,40}$.

A model of stereocilia dynamics in which a zone of rapid turnover is immediately adjacent to a long-lived F-actin core is not without precedence in other eukaryotic structures. ELLPs that elude turnover ${ }^{25}$ are found in the nuclear pore complexes of non-dividing cells. Some nucleoporins have half-lives on the order of months to years, while structurally adjacent nucleoporins encoded by different genes are rapidly replaced ${ }^{25}$. Emerging evidence supports the idea that a protein's half-life is not necessarily uniform throughout the cell or even within the same subcellular compartment. Instead, a protein's stability likely depends on several factors, including posttranslational modifications, interacting partners and subcellular localization $^{41,42}$. Our data are consistent with the view that actin and actin-binding proteins comprising stereocilia cores are ELLPs, though the modifications that allow this long-term persistence remain elusive.

The repair mechanisms of long-lived structures such as stereocilia cores remain to be discovered. For example, gaps in phalloidin-stained F-actin cytoskeletons of stereocilia are particularly abundant after noise trauma or in the absence of either $\beta$ - or $\gamma$-actin ${ }^{14,15}$. Toyama and Hetzer ${ }^{41}$ have suggested that unrepaired damaged ELLPs may contribute to aging processes. Furthermore, some of the homeostatic mechanisms that maintain hair cells and preserve stereocilia function include the secretion of protective molecules from supporting cells and active remodelling of protein complexes that comprise tip links ${ }^{43-45}$. A fuller understanding of homeostasis and repair mechanisms within each of the various stereocilia compartments during aging may have translational potential for preservation and restoration of healthy hearing.

\section{Methods}

Plasmids and biolistic transfection. For live-cell imaging experiments, human ACTB cDNA (NM_001101.3, identical amino acid sequence to mouse $\beta$-actin) cloned into the Green FP expression vector (BD Biosciences, San Jose, CA) was used to prepare gold particles for biolistic transfection as previously described ${ }^{42}$ with minor modifications. Specifically, we used antibiotics in the culture media (see Organ culture and live imaging below) and reduced the BioRad Helios Gene Gun firing pressure to 100 p.s.i. in order to reduce damage caused by biolistic

transfection. The p.G13R and p.G63D substitutions of $\beta$-actin were introduced into 
pEGFP- $\beta$-actin using site-directed mutagenesis (Stratagene, La Jolla, CA) with primers $5^{\prime}$-CGTCGTCGACAACCGCTCCGGCATGTG-3', 5' -CACATGCCGG AGCGGTTGTCGACGACG-3' ${ }^{\prime}$ 5'-CCCAGAGCAAGAGAGACATCCTCA CCCTGAA- $3^{\prime}$ and $5^{\prime}$-TTCAGGGTGAGGATGTCTCTCTTGCTCTGGG-3', respectively (point mutation in bold). pDendra2- $\beta$-actin was cloned using InFusion (Clontech, Mountain View, CA) with primers $5^{\prime}$-GTGTACAAGACTCGAGC CACCATGGATGATGATATCG- ${ }^{\prime}$ and $5^{\prime}$-TAGATCCGGTGGATCCCTAGAAG CATTTGCGGT- $3^{\prime}$ to amplify the cDNA of ACTB and insert it between the XhoI and BamHI sites of pDendra2-C (Clontech, Mountain View, CA). The DNA sequences of all plasmid inserts were verified by Sanger sequencing.

Organ culture and live imaging. C57Bl6/J pups were killed by decapitation at postnatal day 2-5 in accordance with National Institutes of Health Institutional Animal Care and Use Committee-approved guidelines under animal study protocol \#1263. Utricles were dissected in L-15 media (Life Technologies, Carlsbad, CA, USA) and cultured on $2 \mathrm{mg} \mathrm{ml}^{-1}$ collagen matrices in DMEM/F-12 with $20 \mathrm{mM}$ L-glutamine, $7 \%$ fetal bovine serum, and 1 unit per ml penicillin-G. One hour before biolistic transfection, growth media was replaced with antibiotic-free media. Transfections were done using a Helios gene gun and endotoxin-free plasmid DNA (NucleoBond Xtra EF, Macharey-Nagel). Twenty-four hours post transfection, cultures were transferred to phenol-red free media supplemented with penicillin-G and mounted under a platinum and nylon harp ${ }^{46}$ at $37^{\circ} \mathrm{C}$ in a humidified $6 \% \mathrm{CO}_{2}$ atmosphere with the stereocilia facing the coverglass and objective.

Cells transfected with pEGFP- $\beta$-actin were live-imaged on a Zeiss Cell Observer spinning disc microscope ( $\times 63$ plan-apochromat, NA 1.4) for $62-92 \mathrm{~h}$. Z-stack volumes $(138 \times 0.4 \mu \mathrm{m})$ were captured at 1 -h intervals. For photoconversion experiments with pDendra2- $\beta$-actin, transfected cultured cells were imaged for $24 \mathrm{~h}$ and $\mathrm{z}$-stack volumes were captured at $30 \mathrm{~min}$ intervals. Dendra2- $\beta$-actin was converted by exposing a defined region of interest to $405 \mathrm{~nm}$ laser light for $15 \times 1$-ms bursts at $6 \%$ of the total laser power (UltraView, Perkin Elmer, Akron, $\mathrm{OH}$, Laser Module 2). Post-acquisition image analyses were performed using ImageJ (http://imagej.nih.gov/ij/) and Volocity (Perkin Elmer, Akron, OH, USA). For automated analysis of dendra2- $\beta$-actin experiments (Fig. $5 \mathrm{~d}$ ), the red photoconverted populations of dendra2- $\beta$-actin and the green non-photoconverted dendra2- $\beta$-actin cuticular plate were identified mathematically. The distance from the centroid of the red population to the edge of the cuticular plate was measured for each time point.

Fixed-cell imaging. Mutant expression constructs pEGFP- $\beta$-actin ${ }^{\mathrm{G} 63 \mathrm{D}}$ and pEGFP- $\beta$-actin G13R, the wild-type EGFP- $\beta$-actin control and the EGFP-only control were biolistically transfected into cultured utricles from neonatal mice (P2-P5) and fixed in $4 \%$ paraformaldehyde at 4 and $24 \mathrm{~h}$ post transfection for imaging ${ }^{17,18,33}$. After fixation, samples were washed in phosphate-buffered saline, counterstained with rhodamine-conjugated phalloidin and mounted in ProLong Antifade Gold (Life Technologies)

Multi-isotope imaging mass spectrometry. Mouse pups born to normal mothers were switched at birth to nurse from mothers who had been fed for a month or more with food containing ${ }^{15} \mathrm{~N}$-leucine $\left(\sim 1.25 \%\right.$ relative to ${ }^{14} \mathrm{~N}$-leucine). Pups were killed after 4 or 15 days. Utricles were fixed overnight at $4{ }^{\circ} \mathrm{C}$ in $4 \%$ formaldehyde $+10 \%$ glutaraldehyde in cacodylate buffer, then processed for plastic embedding and thin sectioning.

We performed MIMS as described previously 47,48 . Briefly, an accelerated beam of $\mathrm{Cs}^{+}$ions, focused to a $30-\mathrm{nm}$ spot, was scanned across the surface of a thin section, sputtering molecules from the surface. Anionic molecules were accelerated back and analysed with a double sector mass spectrometer. ${ }^{15} \mathrm{~N}$ was detected as the cyanide ion ${ }^{12} \mathrm{C}^{15} \mathrm{~N}^{-}$at mass 27 and compared with ${ }^{12} \mathrm{C}^{14} \mathrm{~N}^{-}$at mass 26 for each pixel in the scanned image. Incorporation was calculated from the natural abundance of ${ }^{15} \mathrm{~N}$ and the relative abundance of ${ }^{15} \mathrm{~N}$ in the food ${ }^{26}$. To show incorporation as a percentage in the image, we used a hue saturation intensity transformation in which the hue represents percentage and the intensity indicates reliability.

\section{References}

1. Nayak, G. D., Ratnayaka, H. S., Goodyear, R. J. \& Richardson, G. P. Development of the hair bundle and mechanotransduction. Int. J. Dev. Biol. 51, 597-608 (2007).

2. Tilney, L. G., Tilney, M. S. \& DeRosier, D. J. Actin filaments, stereocilia, and hair cells: how cells count and measure. Annu. Rev. Cell Biol. 8, 257-274 (1992).

3. Tilney, L. G., Derosier, D. J. \& Mulroy, M. J. The organization of actin filaments in the stereocilia of cochlear hair cells. J. Cell Biol. 86, 244-259 (1980).

4. Kelley, M. W. Cellular commitment and differentiation in the organ of Corti. Int. J. Dev. Biol. 51, 571-583 (2007).

5. Walters, B. J. \& Zuo, J. Postnatal development, maturation and aging in the mouse cochlea and their effects on hair cell regeneration. Hear. Res. 297, 68-83 (2013)
6. Frolenkov, G. I., Belyantseva, I. A., Friedman, T. B. \& Griffith, A. J. Genetic insights into the morphogenesis of inner ear hair cells. Nat. Rev. Genet. 5, 489-498 (2004)

7. Furness, D. N., Mahendrasingam, S., Ohashi, M., Fettiplace, R. \& Hackney, C. $\mathrm{M}$. The dimensions and composition of stereociliary rootlets in mammalian cochlear hair cells: comparison between high- and low-frequency cells and evidence for a connection to the lateral membrane. J. Neurosci. 28, 6342-6353 (2008).

8. Kitajiri, S. et al. Actin-bundling protein TRIOBP forms resilient rootlets of hair cell stereocilia essential for hearing. Cell 141, 786-798 (2010).

9. Daudet, N. \& Lebart, M. C. Transient expression of the t-isoform of plastins/ fimbrin in the stereocilia of developing auditory hair cells. Cell Motil. Cytoskeleton 53, 326-336 (2002).

10. Shin, J. B. et al. The $\mathrm{R} 109 \mathrm{H}$ variant of fascin-2, a developmentally regulated actin crosslinker in hair-cell stereocilia, underlies early-onset hearing loss of DBA/2J mice. J. Neurosci. 30, 9683-9694 (2010).

11. Zheng, L., Beeler, D. M. \& Bartles, J. R. Characterization and regulation of an additional actin-filament-binding site in large isoforms of the stereocilia actin-bundling protein espin. J. Cell Sci. 127, 1306-1317 (2014).

12. Zheng, L. et al. The deaf jerker mouse has a mutation in the gene encoding the espin actin-bundling proteins of hair cell stereocilia and lacks espins. Cell 102, 377-385 (2000)

13. Gorelik, J. et al. Dynamic assembly of surface structures in living cells. Proc. Natl Acad. Sci. USA 100, 5819-5822 (2003).

14. Belyantseva, I. A. et al. Gamma-actin is required for cytoskeletal maintenance but not development. Proc. Natl Acad. Sci. USA 106, 9703-9708 (2009).

15. Perrin, B. J., Sonnemann, K. J. \& Ervasti, J. M. beta-actin and gamma-actin are each dispensable for auditory hair cell development but required for Stereocilia maintenance. PLoS Genet. 6, e1001158 (2010).

16. Di Pasquale, G. et al. A novel bovine virus efficiently transduces inner ear neuroepithelial cells. Mol. Ther. 11, 849-855 (2005).

17. Rzadzinska, A. K., Schneider, M. E., Davies, C., Riordan, G. P. \& Kachar, B. An actin molecular treadmill and myosins maintain stereocilia functional architecture and self-renewal. J. Cell Biol. 164, 887-897 (2004).

18. Schneider, M. E., Belyantseva, I. A., Azevedo, R. B. \& Kachar, B. Rapid renewal of auditory hair bundles. Nature 418, 837-838 (2002).

19. Fujiwara, I., Takahashi, S., Tadakuma, H., Funatsu, T. \& Ishiwata, S. Microscopic analysis of polymerization dynamics with individual actin filaments. Nat. Cell Biol. 4, 666-673 (2002).

20. Kuhn, J. R. \& Pollard, T. D. Real-time measurements of actin filament polymerization by total internal reflection fluorescence microscopy. Biophys. J 88, 1387-1402 (2005).

21. Bugyi, B. \& Carlier, M. F. Control of actin filament treadmilling in cell motility. Annu. Rev. Biophys. 39, 449-470 (2010).

22. Craig, E. M., Van Goor, D., Forscher, P. \& Mogilner, A. Membrane tension, myosin force, and actin turnover maintain actin treadmill in the nerve growth cone. Biophys. J. 102, 1503-1513 (2012).

23. Wegner, A. Head to tail polymerization of actin. J. Mol. Biol. 108, 139-150 (1976).

24. Naoz, M., Manor, U., Sakaguchi, H., Kachar, B. \& Gov, N. S. Protein localization by actin treadmilling and molecular motors regulates stereocilia shape and treadmilling rate. Biophys. J. 95, 5706-5718 (2008).

25. Savas, J. N., Toyama, B. H., Xu, T., Yates, 3rd J. R. \& Hetzer, M. W. Extremely long-lived nuclear pore proteins in the rat brain. Science 335, 942 (2012).

26. Zhang, D. S. et al. Multi-isotope imaging mass spectrometry reveals slow protein turnover in hair-cell stereocilia. Nature 481, 520-524 (2012).

27. Caberlotto, E., Michel, V., de Monvel, J. B. \& Petit, C. Coupling of the mechanotransduction machinery and F-actin polymerization in the cochlear hair bundles. Bioarchitecture 1, 169-174 (2011).

28. Burns, J. C., On, D., Baker, W., Collado, M. S. \& Corwin, J. T. Over half the hair cells in the mouse utricle first appear after birth, with significant numbers originating from early postnatal mitotic production in peripheral and striolar growth zones. J. Assoc. Res. Otolaryngol. 13, 609-627 (2012).

29. Kirkegaard, M. \& Nyengaard, J. R. Stereological study of postnatal development in the mouse utricular macula. J. Comp. Neurol. 492, 132-144 (2005).

30. Noguchi, T. Q. et al. Dominant negative mutant actins identified in flightless Drosophila can be classified into three classes. J. Biol. Chem. 285, 4337-4347 (2010).

31. Posern, G., Sotiropoulos, A. \& Treisman, R. Mutant actins demonstrate a role for unpolymerized actin in control of transcription by serum response factor. Mol. Biol. Cell 13, 4167-4178 (2002).

32. Gurskaya, N. G. et al. Engineering of a monomeric green-to-red photoactivatable fluorescent protein induced by blue light. Nat. Biotechnol. 24, 461-465 (2006).

33. Belyantseva, I. A. Helios Gene Gun-mediated transfection of the inner ear sensory epithelium. Methods Mol. Biol. 493, 103-123 (2009).

34. Salvany, L., Muller, J., Guccione, E. \& Rorth, P. The core and conserved role of MAL is homeostatic regulation of actin levels. Genes Dev. 28, 1048-1053 (2014). 
35. Narayanan, P. et al. Length regulation of mechanosensitive stereocilia depends on very slow actin dynamics and filament-severing proteins. Nat. Commun doi: 10.1038/ncomms7855 (2015).

36. Pollard, T. D. \& Borisy, G. G. Cellular motility driven by assembly and disassembly of actin filaments. Cell 112, 453-465 (2003).

37. Ridley, A. J. Life at the leading edge. Cell 145, 1012-1022 (2011).

38. Halpain, S. Actin in a supporting role. Nat. Neurosci. 6, 101-102 (2003).

39. Sankaranarayanan, S., Atluri, P. P. \& Ryan, T. A. Actin has a molecular scaffolding, not propulsive, role in presynaptic function. Nat. Neurosci. 6, 127-135 (2003).

40. Beurg, M., Fettiplace, R., Nam, J. H. \& Ricci, A. J. Localization of inner hair cell mechanotransducer channels using high-speed calcium imaging. Nat. Neurosci. 12, 553-558 (2009).

41. Toyama, B. H. \& Hetzer, M. W. Protein homeostasis: live long, won't prosper. Nat. Rev. Mol. Cell Biol. 14, 55-61 (2013).

42. Toyama, B. H. et al. Identification of long-lived proteins reveals exceptional stability of essential cellular structures. Cell 154, 971-982 (2013).

43. Indzhykulian, A. A. et al. Molecular remodeling of tip links underlies mechanosensory regeneration in auditory hair cells. PLoS Biol. 11, e1001583 (2013).

44. May, L. A. et al. Inner ear supporting cells protect hair cells by secreting HSP70. J. Clin. Invest. 123, 3577-3587 (2013).

45. Monzack, E. L. \& Cunningham, L. L. Lead roles for supporting actors: critical functions of inner ear supporting cells. Hear. Res. 303, 20-29 (2013).

46. Bird, J. E., Daudet, N., Warchol, M. E. \& Gale, J. E. Supporting cells eliminate dying sensory hair cells to maintain epithelial integrity in the avian inner ear. J. Neurosci. 30, 12545-12556 (2010).

47. Steinhauser, M. L. et al. Multi-isotope imaging mass spectrometry quantifies stem cell division and metabolism. Nature 481, 516-519 (2012).

48. Lechene, C. et al. High-resolution quantitative imaging of mammalian and bacterial cells using stable isotope mass spectrometry. J. Biol. 5, 20 (2006).

\section{Acknowledgements}

We acknowledge Dennis Drayna and Benjamin Perrin for critiques of this manuscript. We thank Lindsey May, Elyssa Monzack, Elizabeth Driver and James Hostetter for assistance and advice in live-cell imaging experiments, Inna Belyantseva for gene gun protocols and Liz Wilson and Barb Zwiesler for help with animal procurement. This work was supported by National Institutes of Health/National Institute of Biomedical Imaging and Bioengineering (NIH/NIBIB) grants P41RR14579 and P41EB001974; NIH grants R01DC00033, R01DC03463, R01DC04179, R37DK39773, R01EY12963, R01GM47214 and R01D K58762; and National Science Foundation Division of Integrative Biology and Neuroscience (NSF/IBN) grant IBN-998298 to C.P.L. D.P.C. is an Investigator of the Howard Hughes Medical Institute. This work was also supported by National Institutes of Health intramural funds from the National Institute on Deafness and Other Communication Disorders DC000079-02 to L.L.C. and DC000039-17 to T.B.F.

\section{Author contributions}

All authors participated in the conception and design of the study; M.C.D., M.B. and D.-S.Z. performed the research; all authors contributed to the analysis of data and wrote the manuscript.

\section{Additional information}

Supplementary Information accompanies this paper at http://www.nature.com/ naturecommunications

Competing financial interests: The authors declare no competing financial interests.

Reprints and permission information is available at http://npg.nature.com/ reprintsandpermissions/

How to cite this article: Drummond, M. C. et al. Live-cell imaging of actin dynamics reveals mechanisms of stereocilia length regulation in the inner ear. Nat. Commun. 6:6873 doi: 10.1038/ncomms7873 (2015).

(c) (i) This work is licensed under a Creative Commons Attribution 4.0 International License. The images or other third party material in this article are included in the article's Creative Commons license, unless indicated otherwise in the credit line; if the material is not included under the Creative Commons license, users will need to obtain permission from the license holder to reproduce the material. To view a copy of this license, visit http://creativecommons.org/licenses/by/4.0/ 